observations included the photographing of Comet $1941 c$ (de Kock), which reached the second magnitude, and of Comet Cunningham, which did not live up to expectations and only just reached naked-eye brightness. The weather at the Cape over the year was cloudier than usual, rainfall being 40 per cent above normal, as the result of considerable falls on a large number of days rather than excessive falls on a few days. Substantial observing losses through cloud were recorded in the programmes of the Victoria telescope and of the photoheliograph, but in spite of the weather the increased efforts of a wardepleted staff actually raised the number of observations made with the transit circle.

\section{A New Telephone Set for Partially Deaf Persons}

An article by A. Herckmans (Bell Lab. Rec., 21, No. 2; October 1942) describes a new amplifying set which recently has been developed for use with the regular telephone set to assist the hard of hearing, and which permits all the added equipment except a small $4 \frac{1}{2}$-volt battery to be incorporated in the base of the telephone. The gain of the amplifier is adjusted by turning one of the switchhook plungers, and the amplifier can be disconnected by a second switch controlled by this same plunger. The switchhook plunger at the right of the set gives gain control and it may be turned to any one of three positions to give low, medium or high gain. Vertical motion of the plunger removes the amplifier from the circuit entirely. The very small amplifier unit consists of a granular-carbon microphone the diaphragm of which is actuated by a bipolar receiver element using permanent magnets. A soft rubber mounting prevents it from picking up vibrations from the surface on which the set is resting. When the amplifier is in use its receiver element is connected in place of the regular handset receiver, while its transmitter element is connected to the handset receiver in series with a $4 \frac{1}{2}$-volt battery which may be placed in any convenient location and connected to the set by two wires. With this arrangement the handset receiver is operated by the amplified speech from the transmitter element of the amplifier.

To prevent 'singing', the amount of gain around the closed path from the receiver to the transmitter through the air path and then back to the receiver through the induction coil must be less than the total loss. A maximum of $25 \mathrm{db}$. gain is provided by the amplifier used and 'singing' will not occur in normal use. A person is not conscious of impairment of hearing for ordinary room conversations until his loss is about $25 \mathrm{db}$., and only about 5 per cent of the population would be conscious of the need for a hearing aid. This percentage would be smaller for telephone conversations because the average speech level received is approximately $10 \mathrm{db}$. higher than that of normal direct conversation. With the additional $25 \mathrm{db}$. provided by the new set, persons with as much as $60 \mathrm{db}$. loss will receive adequate volume. Since only 0.5 per cent have a loss greater than this, the new set provides the help needed for about 90 per cent of those conscious of hearing impairment and would undoubtedly give considerable assistance to many with losses in excess of this.

\section{Application of Electricity in Mine Pumping}

G. B. Alvey and N. Tetlow, in a paper on this subject read before the Institution of Electrical Engineers on December 10, give a good deal of useful information upon electrically driven mine-drainage pumps, which may be of the centrifugal or of the positive displacement reciprocating type. Since the characteristics of the driven pump have a considerable bearing on the choice of the most suitable driving motor, the first portion of the paper deals with operating and design characteristics of the two types of pump. The remainder shows how, by a study of these operating characteristics, the most suitable type of driving motor may be chosen.

\section{Control of Venereal Diseases in Peru}

LAws concerning the medical care of patients suffering from venereal diseases in Peru have recently been promulgated by the President (J.Amer. Med. Assoc., October 17). A national department dealing with these diseases has recently been established with its headquarters in Lima. Treatment during the contagious stage is obligatory. If the patients are minors or mentally deficient, the parents are made responsible for their treatment. Drugs for the treatment of venereal disease are now sold only on medical prescriptions, and the sale of specific medicine by unauthorized persons and treatment by correspondence are prohibited. Wet nurses must have a certificate of health, which is given free of charge in the dispensaries and hospitals. Five per cent of all the beds in public hospitals are to be reserved for venereal diseases.

\section{Edinburgh Geological Society: Clough Memorial Medal and Fund}

THe Clough Memorial Medal for the years 19411942 has been presented to Mr. James L. Begg, of Mount Vernon, Glasgow, for his outstanding contributions to Scottish palæontology. He has worked for many years on the Ordovician rocks of Southern Scotland and has discovered more than a hundred new species of trilobites, mollusca, brachiopods, and other organisms, many of which belong to new genera. The majority of the specimens have been obtained from the classical sections of the Girvan region. Mr. Begg, who is a past president of the Geological Society of Glasgow, has also served as its honorary treasurer for the past twenty-five years.

The Clough Memorial Fund was instituted in 1935 for the purpose of encouraging geological research in Scotland and the north of England. The north of England is defined as comprising the counties of Northumberland, Cumberland, Durham, Westmorland, and Yorkshire. Under the terms of the administration of the fund a sum of approximately $£ 30$ is available annually. Applications for grants are invited for the period April 1, 1943-March 31, 1944. These should be sent to the Secretary, Clough Research Fund Committee, "Edinburgh Geological Society, Synod Hall, Castle Terrace, Edinburgh.

\section{Greetings from Russian Physiologists}

Prow. A. V. HruL, joint secretary of the Royal Society and foreign secretary of the Physiological Society, has received a cable of New Year greetings from the All-Union and Moscow Societies of Physiologists, Pharmacologists and Biochemists, expressing their confidence in complete victory during 1943 for the democratic countries. In his reply, Prof. Hill said he looked forward to joining with thein in holding the Congress of Physiologists deferred from 194I in Great Britain during 1944. 